\title{
MicroRNA-195-5p acts as an anti-oncogene by targeting PHF19 in hepatocellular carcinoma
}

\author{
HUI XU ${ }^{1,2^{*}}$, YAN-WEI HU ${ }^{1 *}$, JIA-YI ZHAO ${ }^{1}$, XIU-MEI HU ${ }^{1}$, SHU-FEN LI $^{1}$, YAN-CHAO WANG ${ }^{1}$, \\ JI-JUAN GAO ${ }^{1}$, YAN-HUA SHA ${ }^{1}$, CHUN-MIN KANG ${ }^{1}$, LI LIN $^{1}$, CHUAN HUANG ${ }^{1}$, \\ JING-JING ZHAO ${ }^{1}$, LEI ZHENG ${ }^{1}$ and QIAN WANG ${ }^{1}$ \\ ${ }^{1}$ Laboratory Medicine Center, Nanfang Hospital, Southern Medical University, Guangzhou, Guangdong 510515; \\ ${ }^{2}$ Hospital of Traditional Chinese Medicine of Qingyuan City, Qingyuan, Guangdong 511500, P.R. China
}

Received January 29, 2015; Accepted April 20, 2015

DOI: $10.3892 /$ or.2015.3957

\begin{abstract}
Hepatocellular carcinoma (HCC) is the fifth most common malignancy worldwide. PHD finger protein 19 (PHF19) encodes a member of the polycomb group (PcG) of proteins that functions by maintaining the repressive transcriptional states of many developmental regulatory genes. In addition, it has been shown that miR-195 plays an important role in the molecular etiology of HCC; however, the effect and possible mechanism of PHF19 on HCC is unclear, and the association between PHF19 and miR-195 has seldom been addressed. In the present study, we investigated the carcinogenic activity and mechanism of PHF19 on HCC in vivo and in vitro. Our results showed that PHF19 is a potential target of hsa-miR-195-5p based on a bioinformatic analysis and results of a luciferase reporter assay. PHF19 was downregulated after transfection with hsa-miR-195-5p mimics. Moreover, we demonstrated that overexpression of PHF19 promoted hepatoma cell migration, invasion and proliferation in vitro. In contrast, overexpression of hsa-miR-195-5p in hepatoma cells reduced PHF19 expression, leading to suppression of hepatoma cell invasion, migration and proliferation in vitro. In addition, PHF19 markedly promoted the growth of xenograft tumors, while hsa-miR-195-5p markedly suppressed the growth of xenograft tumors in nude mice. These results provide evidence that PHF19 promotes HCC and is regulated by the tumor-suppressor, miR-195-5p.
\end{abstract}

Correspondence to: Professor Qian Wang or Professor Lei Zheng, Laboratory Medicine Center, Nanfang Hospital, Southern Medical University, Guangzhou, Guangdong 510515, P.R. China

E-mail: nfyywangqian@163.com

E-mail:nfyyzl@163.com

*Contributed equally

Key words: hepatocellular carcinoma, proliferation, miR-195-5p, PHF19, migration

\section{Introduction}

Hepatocellular carcinoma (HCC) accounts for $70-85 \%$ of primary liver cancers and ranks as the second leading cause of cancer-related deaths among males worldwide (1). HCC usually develops in cirrhotic livers secondary to viral infections [hepatitis B virus (HBV) and hepatitis C virus (HCV)], alcohol abuse, metabolic disorders, or carcinogenic agents $(2,3)$. HCC has heterogeneous etiologic and molecular profiles and varied responses to therapeutics (4). This tumor diversity arises from the multistep hepatocarcinogenic processes requiring sequential genetic and epigenetic alterations, including gene mutations and/or chromosome instability $(3,5,6)$.

PHD finger protein 19 (PHF19), a component of polycomb repressive complex 2 (PRC2), encodes a member of the polycomb group $(\mathrm{PcG})$ of proteins that functions by maintaining repressive transcriptional states of many developmental regulatory genes $(7,8)$. A previous study confirmed that PHF19 is frequently upregulated in several types of cancer (7). Ghislin et al (9) reported that PHF19 silencing reduced the cell proliferation rate and increased the transendothelial migration capacity of melanoma cell lines. Li et al (10) showed that the expression of PHF19 was increased in glioblastoma multiforme samples and correlated positively with astrocytoma grades. These observations suggest that PFH19 is strongly linked to aggressive tumor behavior and are in agreement with increased expression in various human tumor types.

microRNAs (miRNAs) are small non-coding RNAs that control gene expression by modulating stability and/or translation of messenger RNA (mRNA) through interactions with specific sequences located in the coding or untranslated regions (UTRs) (11-13). Several studies have implicated miRNAs in a number of cancers; altered miRNA levels can result in the aberrant expression of gene products that may contribute to carcinogenesis (14-16). Recently, miR-195 was shown to be downregulated in a variety of cancers, including liver, gastric, bladder, breast and adrenocortical cancers (17-20). Introduction of miR-195 markedly suppresses colony formation in vitro and tumor development in nude mice (20). In vitro data suggest that miR-195 suppressed tumorigenicity and regulates the G1/S transition of human HCC cells (17); however, the mechanism underlying miR-195 on tumor development and the association 
between miR-195 and PHF19 and the effect on HCC has not been determined.

In the present study, we demonstrated that PHF19 is a potential target of miR-195-5p based on a bioinformatic analysis and the results of a luciferase reporter assay. Moreover, we demonstrated that overexpression of PHF19 promoted hepatoma cell migration, invasion and proliferation and reduced cell apoptosis in vitro. In contrast, overexpression of hsa-miR-195-5p in hepatoma cells reduced PHF19 expression, leading to inhibition of hepatoma cell invasion, migration and proliferation and promoted cell apoptosis in vitro. In addition, hsa-miR-195-5p markedly suppressed the growth of xenograft tumors in nude mice, while PHF19 promoted the growth of xenograft tumors in nude mice.

\section{Materials and methods}

Sources of tissue samples. Paired HCC and adjacent non-tumor liver tissues were obtained from $30 \mathrm{HCC}$ patients undergoing HCC resection. The specimens were collected between April 2013 and April 2014 in the Department of Infectious Diseases and Institute of Hepatology of Nanfang Hospital. All tissues were confirmed by pathologic examination. Informed consent was obtained from each patient and ethical approval was obtained from the Institutional Review Board of Nanfang Hospital of Southern Medical University.

Cell culture. Human HepG2 cells were obtained from the American Type Culture Collection (ATCC; Manassas, VA, USA) and grown in Dulbecco's modified Eagle's medium (DMEM) containing 10\% fetal calf serum (FCS) with streptomycin $(100 \mu \mathrm{g} / \mathrm{ml})$ and penicillin $(100 \mathrm{U} / \mathrm{ml})$. HepG2 cells were incubated at $37^{\circ} \mathrm{C}$ in $5 \% \mathrm{CO}_{2}$. Cells were seeded in 6- or 12 -well plates or $60-\mathrm{mm}$ dishes and grown to $80-90 \%$ confluency before use.

Lentivirus $(L V)$ production and infection. Cells were cultured in $25 \mathrm{~cm}^{2}$ vented flasks containing DMEM with $10 \%$ FCS under standard culture conditions $\left(37^{\circ} \mathrm{C}\right.$ at $\left.5 \% \mathrm{CO}_{2}\right)$. Packed empty LV vectors with green fluorescent protein (GFP, LV-Mock), LV-mediated human PHF19 overexpression vector (LV-PHF19), and LV-mediated hsa-miR-195-5p overexpression vector (LV-miR-195-5p) with GFP were prepared as previously reported (21). The cells were infected with the LV stock at a multiplicity of infection of 20 transducing units per cell in the presence of $8 \mathrm{mg} / \mathrm{ml}$ of polybrene. Then, cells were washed with fresh complete media after a 24-h incubation. The GFP-positive cells were counted $96 \mathrm{~h}$ post-transduction.

Tumor xenograft experiments. All studies were performed in accordance with protocols approved by the Animal Use and Care Committee of Nanfang Hospital. Athymic nude mice (BALB/c, specific pathogen-free grade, male, $\sim 16 \mathrm{~g}$, 4 week-old) were randomized into 4 groups [LV-Mock $(n=14)$, LV-PHF19 $(\mathrm{n}=14)$, LV-Mock $(\mathrm{n}=12)$ and LV-miR-195-5p $(\mathrm{n}=12)]$ and housed five per cage at $25^{\circ} \mathrm{C}$ under a 12 -h light/dark cycle. All mice were fed with autoclaved mouse chow. The inoculation dosage was $1 \times 10^{7}$ HepG2 cells in $200 \mu \mathrm{l}$ of phosphate-buffered saline per mouse. Mice in the LV-Mock group were injected subcutaneously with control LV (LV-Mock), mice in the LV-PHF19 group were injected subcutaneously with LVs overexpressing PHF19 (LV-PHF19) and mice in the LV-miR-195-5p group were injected subcutaneously with LVs overexpressing hsa-miR-195-5p (LV-miR-195-5p). The body weights of all of nude mice and metastatic tumors were measured every 3 days. All mice were sacrificed and the formed tumors were removed.

RNA isolation and real-time quantitative PCR analysis. Total RNA from liver tissues was extracted using TRIzol reagent (Invitrogen, Carlsbad, CA, USA) in accordance with the manufacturer's instructions. The mRNA levels were determined by real-time quantitative PCR using an ABI 7500 Fast Real-Time PCR system with SYBR-Green Detection chemistry (Takara Bio, Inc., Shiga, Japan). The expression of GAPDH was used as the internal control. The miRNA levels were determined by real-time quantitative PCR using an ABI 7500 Fast Real-Time PCR system with the All-in-One ${ }^{\mathrm{TM}}$ miRNA qPCR kit in 20- $\mu 1$ reaction volumes (GeneCopoeia, Rockville, MD, USA) according to the instruction manual. The expression of U6 RNA was used as an endogenous control. Quantitative measurements were determined using the $\Delta \Delta \mathrm{Ct}$ method. All samples were measured in triplicate and the mean value was considered for comparative analysis.

Western blot analyses. Cells were harvested and protein extracts prepared according to established methods. Extracts were then separated by $10 \%$ sodium dodecyl sulfate-polyacrylamide gel electrophoresis and subjected to western blot analyses using rabbit polyclonal anti-PHF19 and rabbit polyclonal anti- $\beta$-actin antibodies (both from Abcam, Cambridge, MA, USA). The proteins were visualized using a chemiluminescence method (ECL Plus Western Blot Detection system; Amersham Biosciences, Foster City, CA, USA).

Transfection of miRNA mimics. HepG2 cells were transfected with $50 \mathrm{nM}$ hsa-miR-195-5p mimics utilizing Lipofectamine 2000 transfection reagent (Invitrogen) for $48 \mathrm{~h}$ according to the manufacturer's instructions. All experimental control samples were treated with an equal concentration of a non-targeting control mimic sequence (negative controls).

Luciferase assay. Human PHF19 cDNA, containing putative and mutant target sites for hsa-miR-195-5p, was chemically synthesized and inserted into a pMIR-REPORT ${ }^{\mathrm{TM}}$ vector (Ambion). The pMIR-REPORT ${ }^{\mathrm{TM}} \beta$-galactosidase control vector (Ambion) was used as a reference. For the luciferase assay, 293T cells (human embryonic kidney 293 cells) were co-transfected with wild- (pMIR-PHF19-wt) or mutant-type (pMIR-PHF19-mt) reporter vectors and hsa-miR-195-5p mimics using Lipofectamine 2000 transfection reagent. Luciferase activity was measured $48 \mathrm{~h}$ post-transfection using a Dual-Luciferase Assay kit (Promega, Madison, WI, USA).

Cell cycle and apoptosis assays. The cells were harvested and cell cycle analysis and apoptosis quantification were performed by flow cytometry using a FACScan flow cytometer (Becton Dickinson Immunocytometry Systems, San Jose, CA, USA) equipped with an argon ion laser (488 $\mathrm{nm}$ ). Cell cycle analysis was performed by flow cytometry using 
a propidium iodide (PI) cell cycle detection kit (Beyotime Institute of Biotechnology, Beijing, China) according to the manufacturer's instructions. Cell apoptosis analysis was performed by flow cytometry using the fluorescein isothiocyanate Annexin V (AV) Apoptosis Detection kit I (BD Biosciences, Bedford, MA, USA) according to the manufacturer's instructions.

Cell proliferation assay. A Cell Counting Kit-8 (CCK-8; Wako Pure Chemical Industries, Osaka, Japan) was used to measure cell viability to evaluate cell proliferation. Cells treated with LV-Mock, LV-PHF19 and LV-miR-195-5p were seeded into 96-well culture plates in triplicate. At various time-points $(0,12,24,36$ and $48 \mathrm{~h}), 10 \mu \mathrm{l}$ of a CCK-8 solution was added to each well in an assay plate and incubated in a $\mathrm{CO}_{2}$ incubator for $1 \mathrm{~h}$. Then, $10 \mu \mathrm{l}$ of $1 \%$ (w/v) SDS solution was added to each well to stop the reaction and the plate was read by a microplate reader for optical density at $450 \mathrm{~nm}$ (Thermo Electron Corporation, Marietta, OH, USA).

Transwell assay. Transwell chambers (Millipore-Chemicon, Billerica, MA, USA) were used for the invasion and migration assays. For the invasion assays, Matrigel-coated chambers were placed in empty wells of 24-well tissue culture plates. Cells were cultured in a serum-free medium for $24 \mathrm{~h}$, then trypsinized and suspended with a culture medium containing $0.1 \%$ bovine serum albumin at a concentration of $5 \times 10^{4}$ cells $/ \mathrm{ml}$. Then, $500 \mu 1$ of the cell suspension was added to each chamber and $750 \mu \mathrm{l}$ of complete medium was added to each well. After incubation for $36 \mathrm{~h}$, the non-invading cells were removed from the upper surface of the chamber membrane using cotton-tipped swabs. The cells on the lower surface of the chamber membrane were fixed in $4 \%$ paraformaldehyde for $10 \mathrm{~min}$, stained with crystal violet, then counted after photographing the membrane through a microscope. For the migration assays, non-coated chambers were used and migrated cells on the lower surface of the chamber membrane were fixed after a 24-h incubation, stained and photographed for further analysis.

Statistical analysis. Data are expressed as the mean \pm standard deviation (SD). The results were analyzed by one-way analysis of variance, followed by the Student-Newman-Keuls (SNK) test and the Student's t-test using SPSS v13.0 statistical software (SPSS, Inc., Chicago, IL, USA). A two-tailed probability $(\mathrm{P})$-value $<0.05$ was considered statistically significant.

\section{Results}

Expression of PHF19 is frequently induced in HCC tissues. In a previous mRNA microarray analysis, we showed that PHF19 was upregulated in HCC tissues compared with adjacent non-tumor tissues. To further confirm and extend this finding, we determined the expression of PHF19 in 30 paired HCC and adjacent non-tumor tissues using real-time PCR analysis. As shown in Fig. 1, the level of PHF19 mRNA was increased in the HCC tissues compared to that in the adjacent non-tumor tissues. These results suggest that induced PHF19 expression is a frequent event in human HCC and may be involved in hepatocarcinogenesis.

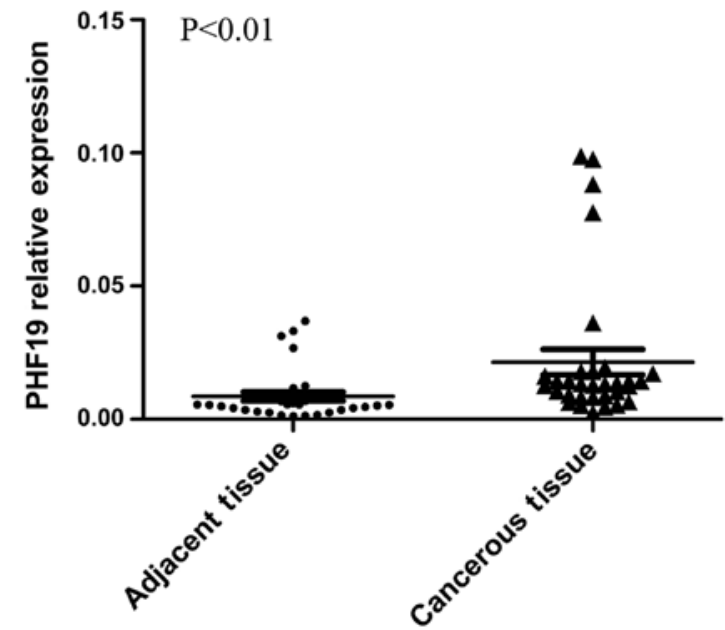

Figure 1. PHF19 gene expression in HCC and adjacent tissues was measured by real-time quantitative PCR. The results are expressed as the mean $\pm \mathrm{SD}$. Each sample was performed at least in triplicate. $\mathrm{P}<0.01$ vs. adjacent tissues. PHF19, PHD finger protein 19; HCC, hepatocellular carcinoma.

PHF19 promotes cell migration, invasion and proliferation, while suppressing apoptosis in vitro. To investigate the biological role of PHF19 in HCC cells, we analyzed the effect of PHF19 on the migration and invasive capacities in HepG2 cells. HepG2 cells were infected with empty LV vectors (LV-Mock) or human PHF19 overexpression LV vectors (LV-PHF19). The expression of PHF19 protein in the HepG2 cells was assessed by western blot analyses. As shown in Fig. 2A, compared to the LV-Mock-treated cells, the expression of PHF19 was markedly increased in the LV-PHF19-treated cells. The Transwell assay was used to determine the effect of PHF19 on HepG2 cell migration and invasion. HepG2 cells infected with LV-PHF19 exhibited significantly increased migration and invasion abilities compared to HepG2 cells infected with LV-Mock (Fig. 2B). Next, we determined whether or not the expression of PHF19 influenced HepG2 cell survival or proliferation in vitro using the CCK-8 assay (Fig. 2C), which showed that PHF19 LV constructs markedly increased the proportion of living HepG2 cells. We further investigated the effects of PHF19 on the cell cycle via flow cytometry. As shown in Fig. 2D, there was a decrease in the percentage of cells in the G1 phase, an increase in the percentage of cells in the $S$ phase, and no change in the percentage of cells in the G2/M phase post-infection compared to the control cells. Apoptosis in the HepG2 cells expressing the LV-Mock and LV-PHF19 vectors was evaluated using co-labeling with AV and PI via flow cytometry. As shown in Fig. 2E, treatment with the LV-PHF19 vector significantly decreased the percentage of apoptotic cells compared to treatment with the LV-Mock vector in the HepG2 cells. Together, these data strongly suggest an important pathologic role for PHF19 in HCC.

Identification of endogenous miR-195-5p controlling PHF19 regulation in liver cancer. It has been shown that all of the known processes involved in cancer, including apoptosis, proliferation, survival and metastasis, are regulated by small regulatory non-coding RNAs consisting of 19-25 nucleotides (i.e., miRNAs). Since PHF19 is upregulated in HCC, we 
A

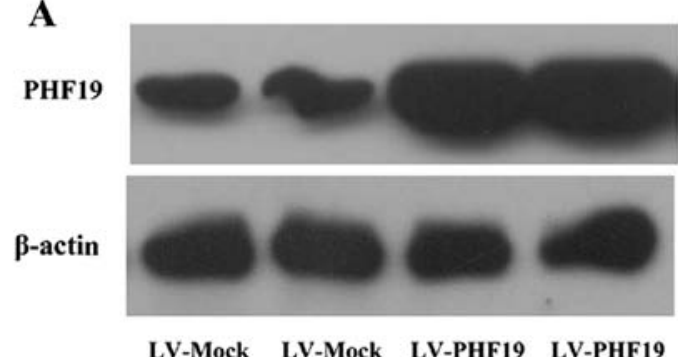

LV-Mock LV-Mock LV-PHF19 LV-PHF19
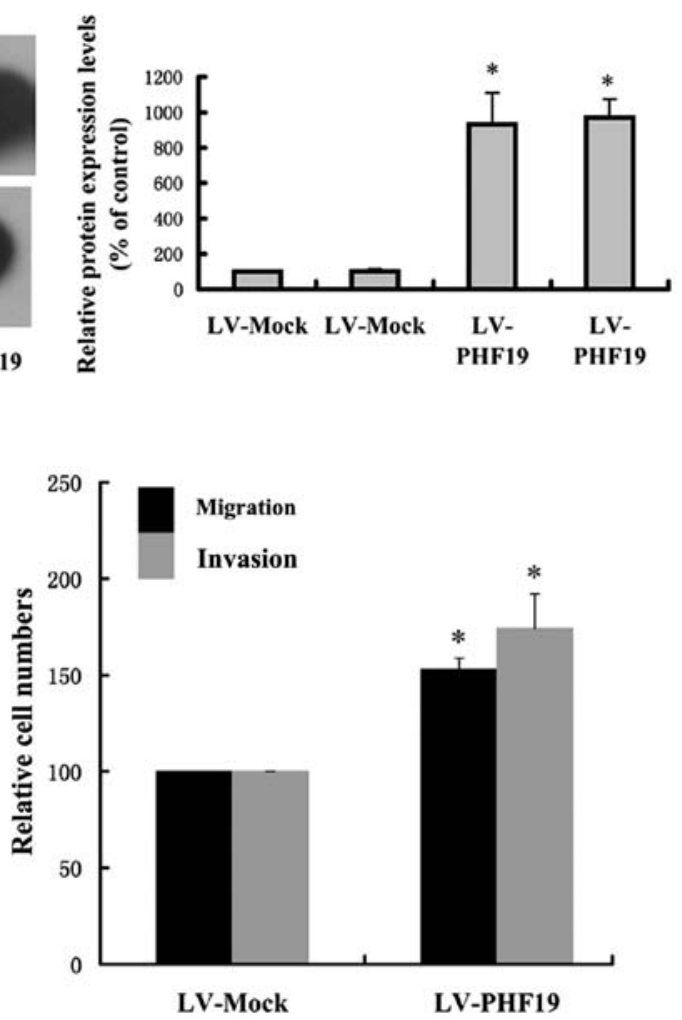
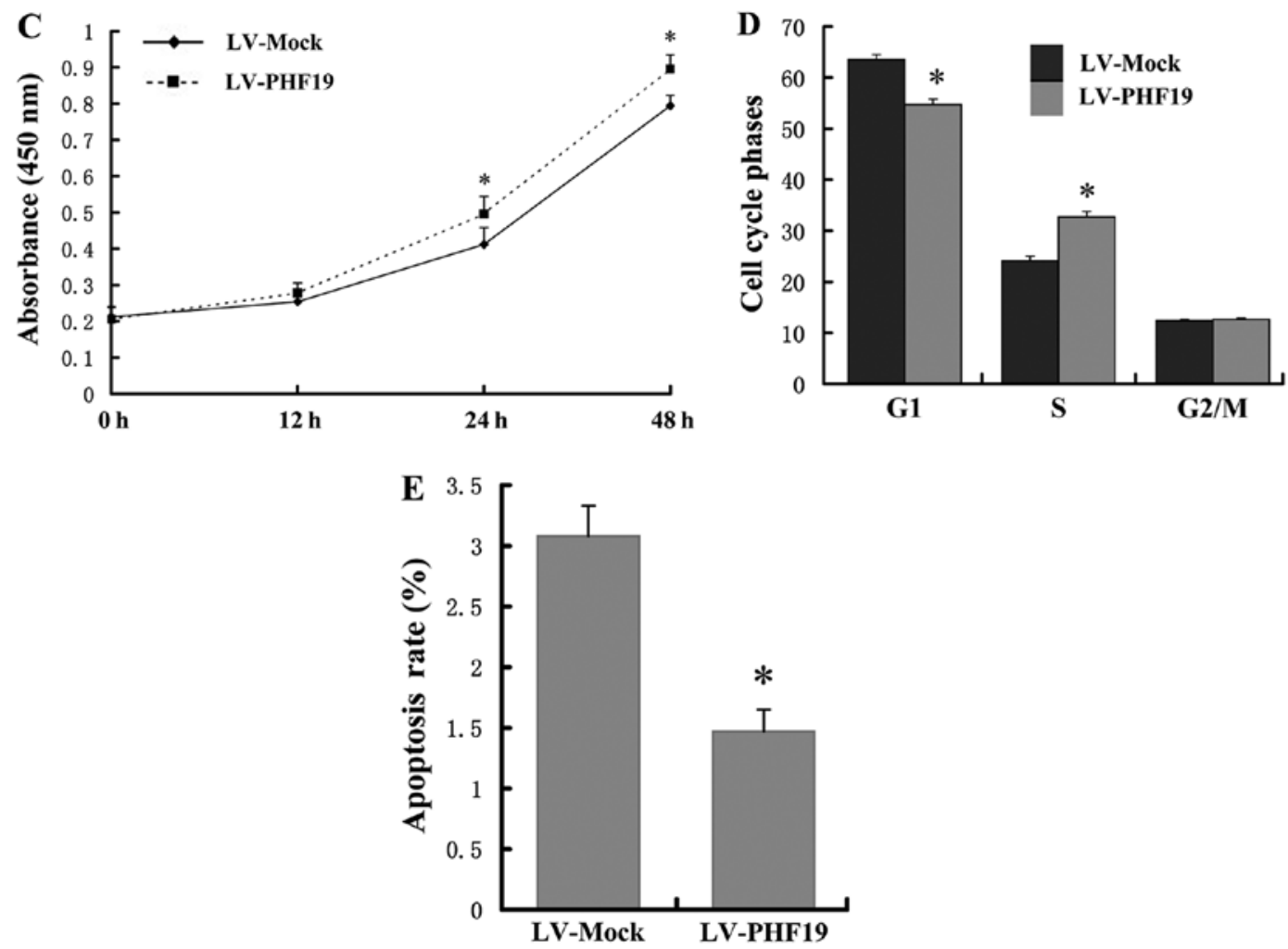

Figure 2. Effects of PHF19 on invasion, migration, proliferation and apoptosis of HepG2 cells. HepG2 cells were treated as indicated and (A) PHF19 protein expression was measured by western blot analysis. (B) Transwell assays were performed to assess migration and invasion by analyzing the number of cells on the Transwell membrane. Cells in five randomlyselected fields were counted. Representative images are shown (magnification, 40x). (C) The CCK-8 cell proliferation assay was performed. (D) The cell cycle distribution was assessed via flow cytometry. (E) The proportion of apoptotic cells was assessed by flow cytometry (50,000 cells counted). $\mathrm{AV}^{+} \mathrm{PI}^{-}$was defined as early apoptosis. The results are expressed as the mean $\pm \mathrm{SD}$ of three independent experiments, each performed in triplicate. " $\mathrm{P}<0.05$ vs. LV-Mock group. PHF19, PHD finger protein 19; AV, Annexin V; PI, propidium iodide; LV, lentivirus.

hypothesized that the expression of PHF19 is balanced by endogenous miRNAs that control PHF19 mRNA translation in normal hepatic liver cells. Loss or suppression of miRNAs targeting PHF19 may cause aberrant overexpression of PHF19, 

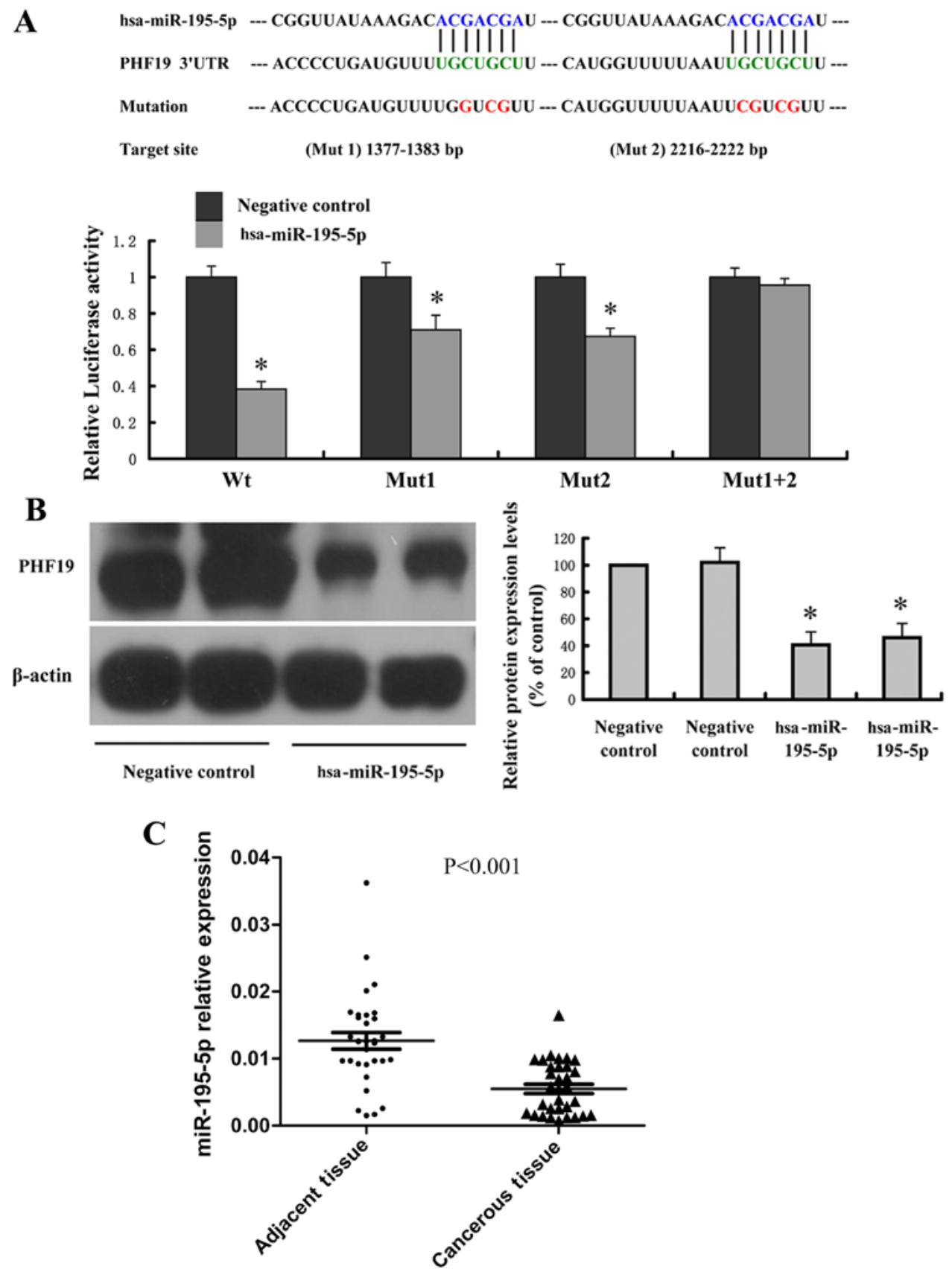

Figure 3. Effects of miR-195-5p on PHF19 expression. (A) Schematic representation of the predicted target regions of miR-195-5p in the 3'UTR of PHF19 and the mutated sequences of these target regions. (B) The protein level of PHF19 was analyzed by western blotting after treatment with miR-195-5p mimics or negative control. The results are expressed as the mean \pm SD of three independent experiments, each performed in triplicate. (C) Western blot analysis of PHF19 protein expression in HepG2 cells treated as indicated. The results are expressed as the mean \pm SD of three independent experiments, each performed in triplicate. ${ }^{\mathrm{P}}<0.05$ vs. negative control group. PHF19, PHD finger protein 19; UTR, untranslated region.

thereby conferring oncogenic potential for hepatocellular malignant proliferation and transformation. We identified a putative miRNA, designated miR-195-5p, which targets human PHF19, utilizing a combination of TargetScan, miRanda, RNA22, miRDB, miR-Gen, PITA, EMBL-EBI, starBase, PicTar, RNAhybrid, and miRBase. The sequence and seed pairing of this miRNA are conserved across multiple species. To establish the effect of miR-195-5p on the 3'UTR of human PHF19, the dual-luciferase assay was performed. As shown in Fig. 3A, human PHF19 mRNA contains two putative complementary sequences to miR-195-5p. Upon co-transfection of 293T cells with wild-type (pMIR-PHF19-wt) reporter vectors and hsa-miR-195-5p using Lipofectamine 2000 transfection reagent, luciferase activity was significantly decreased. Mutation of binding site 1 or 2 markedly suppressed the effect of hsa-miR-195-5p, while mutation of both binding sites completely reversed the effect of hsa-miR-195-5p. Consistent with the results of the luciferase assays, mimics of miR-195-5p were able to suppress endogenous PHF19 protein expression (Fig. 3B). In addition, we determined the levels of miR-195-5p in 30 paired HCC and adjacent non-tumor tissues by real-time PCR analysis. As shown in Fig. 3C, the level of miR-195-5p was decreased in the HCC tissues compared to adjacent non-tumor tissues. 
$\mathbf{A}$
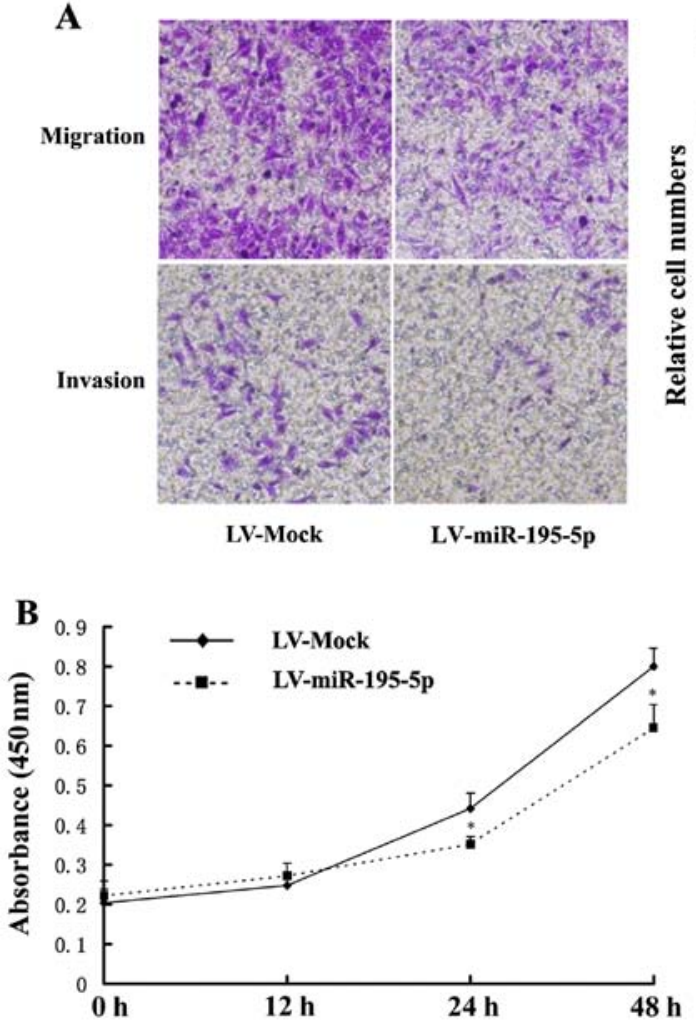
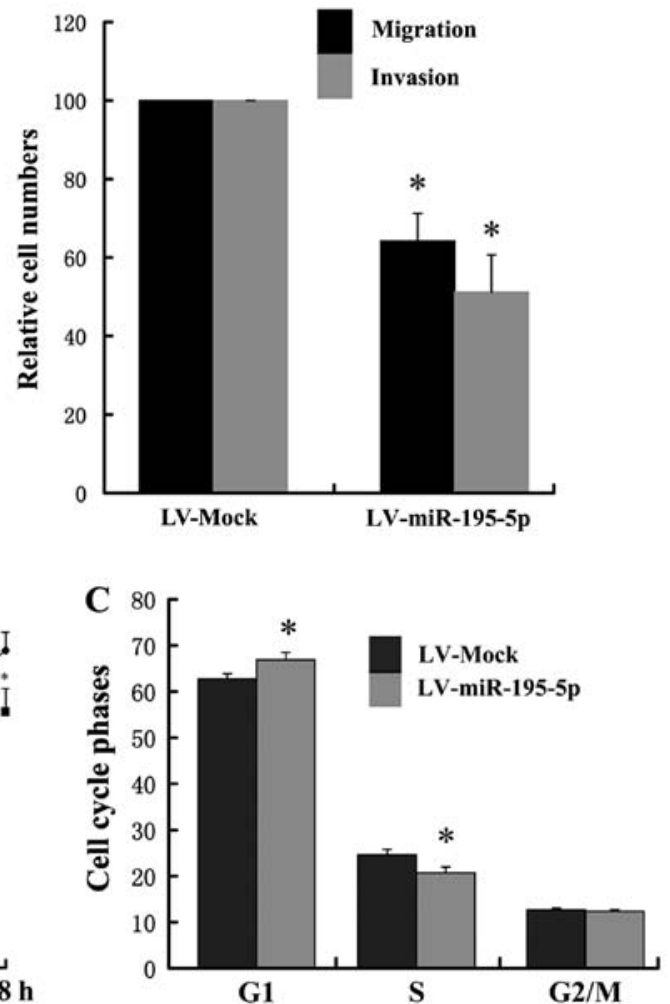

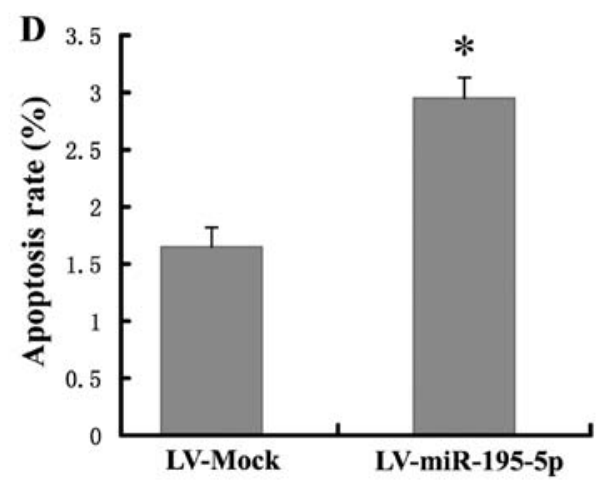

Figure 4. Effects of hsa-miR-195-5p on invasion, migration, proliferation and apoptosis of HepG2 cells. HepG2 cells were treated as indicated and (A) Transwell assays were performed to assess migration and invasion by analyzing the number of cells on the Transwell membrane. Cells in five randomly selected fields were counted. Representative images are shown (magnification, 40x). Data are presented as the mean \pm SD of three independent experiments. (B) The CCK- 8 cell proliferation assay was performed. (C) The cell cycle distribution was assessed via flow cytometry. (D) The proportion of apoptotic cells was assessed by flow cytometry $\left(50,000\right.$ cells counted). $\mathrm{AV}^{+} \mathrm{PI}-$ was defined as early apoptosis. The results are expressed as the mean $\pm \mathrm{SD}$ of three independent experiments, each performed in triplicate. ${ }^{*} \mathrm{P}<0.05$ vs. LV-Mock group. AV, Annexin V; PI, propidium iodide; LV, lentivirus.

miR-195-5p suppresses cell invasion, migration and proliferation, while promoting apoptosis in vitro. Since PHF19 is a target for hsa-miR-195-5p and takes part in regulating invasion, migration, proliferation and apoptosis of HepG2 cells, we next investigated the effects of hsa-miR-195-5p on invasion, migration, proliferation and apoptosis in the HepG2 cells. HepG2 cells were infected with empty LV vectors (LV-Mock) or hsa-miR-195-5p overexpression LV vectors (LV-miR-195-5p). As shown in Fig. 4A, Transwell assays revealed that LV-miR195-5p-infected HepG2 cells displayed a significantly decreased ability to migrate and invade compared to the LV-Mock-infected HepG2 cells. Moreover, the CCK-8 assay showed that LV-miR-195-5p treatment significantly decreased the proportion of living HepG2 cells (Fig. 4B). As shown in Fig. 4C, LV-miR-195-5p-infected HepG2 cells showed a decrease in the percentage of cells in the G1 phase, an increase in the percentage of cells in the $S$ phase, and no change in the percentage of cells in the $\mathrm{G} 2 / \mathrm{M}$ phase compared to the control cells. As shown in Fig. 4D, LV-miR-195-5p significantly induced the HepG2 cell apoptosis rate. These findings indicate that hsa-miR-195-5p may suppress HepG2 cell invasion, migration and proliferation, while inducing apoptosis through inhibition of PHF19 expression.

PHF19 promotes the tumorigenicity of HCC, while miR195-5p suppresses the tumorigenicity of HCC cells in nude mice. To validate the effect of PHF19 and miR-195-5p on HCC in vivo, HepG2 cells infected with an adenovirus expressing PHF19 (LV-PHF19), miR-195-5p (LV-miR-195-5p) or control adenovirus (LV-Mock) were injected subcutaneously into 

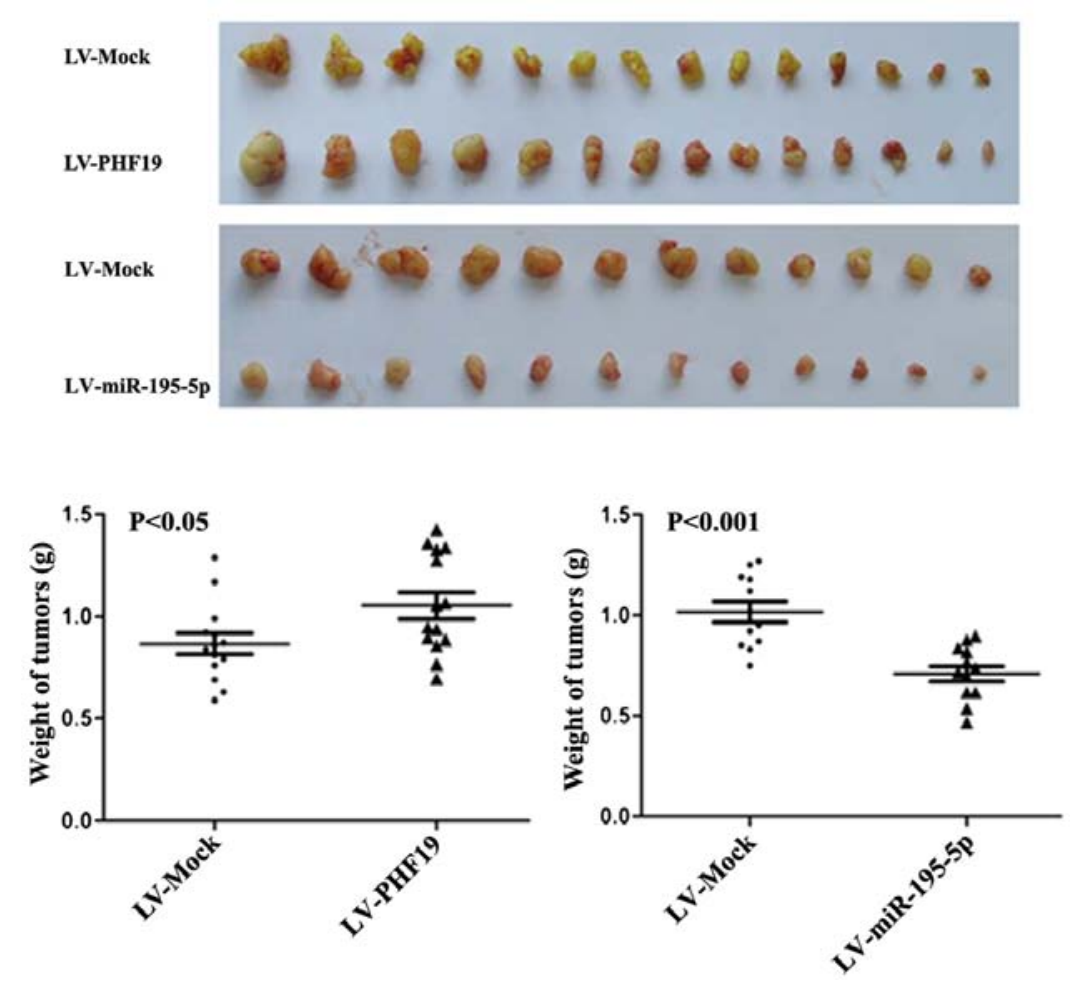

Figure 5. Effects of PHF19 and hsa-miR-195-5p on xenograft tumors. HepG2 cells infected with LV-PHF19, LV-miR-195-5p or LV-Mock were injected subcutaneously into the nude mice. Representative tumors were removed and photographed. PHF19, PHD finger protein 19; LV, lentivirus.

nude mice. Four weeks after the subcutaneous injections, the tumors were removed and weighed. As shown in Fig. 5, tumor growth was promoted in the mice injected with LV-PHF19treated HepG2 cells and reduced in the mice injected with the LV-miR-195-5p-treated HepG2 cells compared to mice injected with LV-Mock-treated HepG2 cells. These results suggest that PHF19 promotes HCC tumorigenicity, while miR195-5p acts as an HCC suppressor.

\section{Discussion}

The global incidence of $\mathrm{HCC}$ is currently estimated at nearly 750,000 new cases each year, resulting in more than 600,000 deaths annually; and the number of deaths is increasing. Patients are typically diagnosed with late-stage disease, leading to poor survival rates (22). The poor prognosis and high recurrence rate of HCC is largely due to the high rate of metastases and malignant proliferation (1). The control of HCC proliferation, apoptosis, invasion and metastasis is of critical importance in the pathogenesis of $\mathrm{HCC}$, as suppression of HCC tumorigenesis leads to reversal of HCC progression. Thus, it is reasonable to explore new strategies through which to suppress the malignant characteristics of HCCs, which may be beneficial in restoring $\mathrm{HCC}$ development. In the present study, we showed that PHF19 is a potential target of hsa-miR195-5p. Overexpression of PHF19 promoted hepatoma cell migration, invasion and proliferation, while overexpression of hsa-miR-195-5p inhibited hepatoma cell invasion, migration and proliferation. In addition, PHF19 promoted the growth of xenograft tumors, while hsa-miR-195-5p suppressed the growth of xenograft tumors in nude mice.
PcG proteins function to maintain the stable epigenetic repression of homeotic genes and other important developmental and cell cycle regulatory genes. Such maintenance establishes a form of cellular memory for its identity or state of differentiation. Accumulating evidence indicates that perturbation of this transcriptional memory may be required for tumor progression and may represent a hallmark of cancer (7). A previous study revealed that PRC2 catalyzes the methylation of histone $\mathrm{H} 3$ at lysine 27 to repress gene expression. PcG-mediated chromatin modification exerts a major influence on the maintaining of gene expression patterns of different cells that are set during early development and differentiation (23-25). As polycomb proteins are involved in the epigenetic control of gene expression and emerge as an attractive therapeutic target for the treatment of cancer, PHF19, as a component of the PRC2, has already attracted increased attention in carcinogenesis. Brien et al (26) demonstrated that depletion of PHF19 leads to proliferative defects, due at least in part to a loss of polycomb binding at and de-repression of the tumor-suppressor p16INK4A in normal fibroblasts and SNF5deficient malignant rhabdoid sarcoma cells. Ectopic expression of PCL3/PHF19 leads to p16INK4A repression and cellular senescence is bypassed. This correlates with increased recruitment of EZH2 and accumulation of H3K27me3 and PRC1 components on the INK4A promoter, suggesting a potential oncogenic role for PHF19 (26). In addition, Callahan et al (27) showed that PHF19 increased HC11 mouse mammary epithelial cell invasion capability. PHF19 was shown to confer properties associated with malignant transformation in HC11 mammary epithelial cells (27). In the present study, PHF19 was increased in the HCC tissues compared to that in the 
adjacent non-tumor tissues. In addition, ectopic expression of PHF19 promoted HCC migration, invasion and proliferation, while suppressing apoptosis in vitro.

Previous studies have shown that miR-195 is frequently downregulated in HCC tissues and cell lines (17). Moreover, gain- and loss-of-function studies revealed that miR-195 can block the human HCC G1/S transition, promote cell proliferation and facilitate the development of HCC (17). In agreement with previous studies, our study demonstrated that the miR-195-5p level was decreased in HCC tissues compared to adjacent non-tumor tissues and miR-195-5p suppressed HepG2 cell invasion, migration and proliferation, while promoting apoptosis. Moreover, our data indicated that the 3'UTR of PHF19 contained two highly-conserved binding sites for hsa-miR-195-5p and the expression of PHF19 mRNA and protein was strongly repressed by hsa-miR-195-5p overexpression in the HepG2 cells. In addition, our orthotopic xenograft experiments revealed that overexpression of PHF19 promoted tumor growth, while overexpression of hsa-miR195-5p suppressed tumor growth, which is consistent with our in vitro findings, demonstrating that PHF19 can promote HCC growth and hsa-miR-195-5p has antitumor activity. These data suggest that PHF19 is regulated by hsa-miR-195-5p.

In conclusion, we provide strong evidence that PHF19 promotes HCC and is regulated by the tumor-suppressor, miR-195-5p. Our data suggest an important role for PHF19 and $\mathrm{miR}-195-5 \mathrm{p}$ in the molecular etiology of cancer and implicate its potential application in HCC therapy; however, more experimental evidence is needed to elucidate the mechanism of PHF19 in HCC.

\section{Acknowledgements}

This study was supported by the Project of Guangdong Provincial Department of Science and Technology (no. 2013B021800086).

\section{References}

1. Parpart S, Roessler S, Dong F, Rao V, Takai A, Ji J, Qin LX, Ye QH, Jia HL, Tang ZY, et al: Modulation of miR-29 expression by $\alpha$-fetoprotein is linked to the hepatocellular carcinoma epigenome. Hepatology 60: 872-883, 2014.

2. Jain S, Singhal S, Lee P and Xu R: Molecular genetics of hepatocellular neoplasia. Am J Transl Res 2: 105-118, 2010.

3. Nault JC and Zucman-Rossi J: Genetics of hepatobiliary carcinogenesis. Semin Liver Dis 31: 173-187, 2011.

4. Li L, Liu Y, Guo Y, Liu B, Zhao Y, Li P, Song F, Zheng H, Yu J, Song T, et al: Regulatory miR-148a-ACVR1/BMP circuit defines a cancer stem cell-like aggressive subtype of hepatocellular carcinoma. Hepatology 61: 574-584, 2015.

5. Boyault S, Rickman DS, de Reyniès A, Balabaud C, Rebouissou S, Jeannot E, Hérault A, Saric J, Belghiti J, Franco D, et al: Transcriptome classification of HCC is related to gene alterations and to new therapeutic targets. Hepatology 45: 42-52, 2007.

6. Hatziapostolou M, Polytarchou C, Aggelidou E, Drakaki A, Poultsides GA, Jaeger SA, Ogata H, Karin M, Struhl K, Hadzopoulou-Cladaras M, et al: An HNF4 $\alpha$-miRNA inflammatory feedback circuit regulates hepatocellular oncogenesis. Cell 147: 1233-1247, 2011.

7. Wang S, Robertson GP and Zhu J: A novel human homologue of Drosophila polycomb like gene is up-regulated in multiple cancers. Gene 343: 69-78, 2004

8. Ballaré C, Lange M, Lapinaite A, Martin GM, Morey L, Pascual G, Liefke R, Simon B, Shi Y, Gozani O, et al: Phf19 links methylated Lys36 of histone $\mathrm{H} 3$ to regulation of Polycomb activity. Nat Struct Mol Biol 19: 1257-1265, 2012.
9. Ghislin S, Deshayes F, Middendorp S, Boggetto N and Alcaide-Loridan C: PHF19 and Akt control the switch between proliferative and invasive states in melanoma. Cell Cycle 11: 1634-1645, 2012

10. Li G, Warden C, Zou Z, Neman J, Krueger JS, Jain A, Jandial R and Chen M: Altered expression of polycomb group genes in glioblastoma multiforme. PLoS One 8: e80970, 2013.

11. Krol J, Loedige I and Filipowicz W: The widespread regulation of microRNA biogenesis, function and decay. Nat Rev Genet 11: 597-610, 2010.

12. Laloo B, Simon D, Veillat V, Lauzel D, Guyonnet-Duperat V, Moreau-Gaudry F, Sagliocco F and Grosset C: Analysis of post-transcriptional regulations by a functional, integrated, and quantitative method. Mol Cell Proteomics 8: 1777-1788, 2009.

13. Breving $\mathrm{K}$ and Esquela-Kerscher A: The complexities of microRNA regulation: mirandering around the rules. Int $\mathrm{J}$ Biochem Cell Biol 42: 1316-1329, 2010.

14. Ji J, Yamashita T, Budhu A, Forgues M, Jia HL, Li C, Deng C, Wauthier E, Reid LM, Ye QH, et al: Identification of microRNA-181 by genome-wide screening as a critical player in EpCAM-positive hepatic cancer stem cells. Hepatology 50: 472-480, 2009

15. Foekens JA, Sieuwerts AM, Smid M, Look MP, de Weerd V, Boersma AW, Klijn JG, Wiemer EA and Martens JW: Four miRNAs associated with aggressiveness of lymph node-negative, estrogen receptor-positive human breast cancer. Proc Natl Acad Sci USA 105: 13021-13026, 2008.

16. Nagel R, le Sage C, Diosdado B, van der Waal $M$, Oude Vrielink JA, Bolijn A, Meijer GA and Agami R: Regulation of the adenomatous polyposis coli gene by the miR-135 family in colorectal cancer. Cancer Res 68: 5795-5802, 2008.

17. Xu T, Zhu Y, Xiong Y, Ge YY, Yun JP and Zhuang SM: microRNA-195 suppresses tumorigenicity and regulates G1/S transition of human hepatocellular carcinoma cells Hepatology 50: 113-121, 2009.

18. Özata DM, Caramuta S, Velázquez-Fernández D, Akçakaya P, Xie H, Höög A, Zedenius J, Bäckdahl M, Larsson C and Lui WO: The role of microRNA deregulation in the pathogenesis of adrenocortical carcinoma. Endocr Relat Cancer 18: 643-655, 2011.

19. Zhang QQ, Xu H, Huang MB, Ma LM, Huang QJ, Yao Q, Zhou H and Qu LH: MicroRNA-195 plays a tumor-suppressor role in human glioblastoma cells by targeting signaling pathways involved in cellular proliferation and invasion. Neuro Oncol 14: 278-287, 2012.

20. Li D, Zhao Y, Liu C, Chen X, Qi Y, Jiang Y, Zou C, Zhang X, Liu S, Wang X, et al: Analysis of miR-195 and miR-497 expression, regulation and role in breast cancer. Clin Cancer Res 17: 1722-1730, 2011.

21. Jin Q, Marsh J, Cornetta K and Alkhatib G: Resistance to human immunodeficiency virus type 1 (HIV-1) generated by lentivirus vector-mediated delivery of the CCR5\{Delta\}32 gene despite detectable expression of the HIV-1 co-receptors. J Gen Virol 89: 2611-2621, 2008

22. Jemal A, Bray F, Center MM, Ferlay J, Ward E and Forman D: Global cancer statistics. CA Cancer J Clin 61: 69-90, 2011.

23. Margueron R and Reinberg D: The Polycomb complex PRC2 and its mark in life. Nature 469: 343-349, 2011.

24. Schuettengruber B and Cavalli G: Recruitment of polycomb group complexes and their role in the dynamic regulation of cell fate choice. Development 136: 3531-3542, 2009.

25. Simon JA and Kingston RE: Mechanisms of polycomb gene silencing: knowns and unknowns. Nat Rev Mol Cell Biol 10: 697-708, 2009.

26. Brien GL, Gambero G, O'Connell DJ, Jerman E, Turner SA, Egan CM, Dunne EJ, Jurgens MC, Wynne K, Piao L, et al: Polycomb PHF19 binds H3K36me3 and recruits PRC2 and demethylase NO66 to embryonic stem cell genes during differentiation. Nat Struct Mol Biol 19: 1273-1281, 2012.

27. Callahan R, Mudunur U, Bargo S, Raafat A, McCurdy D, Boulanger C, Lowther W, Stephens R, Luke BT, Stewart C, et al: Genes affected by mouse mammary tumor virus (MMTV) proviral insertions in mouse mammary tumors are deregulated or mutated in primary human mammary tumors. Oncotarget 3 : 1320-1334, 2012. 